\title{
Avaliação da Vitalidade Fetal em Gestantes Diabéticas: Análise dos Resultados Neonatais
}

\author{
Fetal Surveillance in Pregnancies Complicated by Diabetes: Analysis of Neonatal Outcome \\ Roseli Mieko Yamamoto, Rossana Pulcineli Vieira Francisco, Seizo Miyadahira \\ Jorge D. Banduki Neto, Marcelo Zugaib
}

\begin{abstract}
RESUMO
Objetivos: estudar os testes de avaliação da vitalidade fetal em gestantes diabéticas e relacionar com os resultados neonatais.

Métodos: estudamos 387 gestantes diabéticas atendidas no Setor de Vitalidade Fetal. O último exame (cardiotocografia, perfil biofisico fetal, indice de liquido amniótico e dopplervelocimetria) foi relacionado com os resultados neonatais.

Resultados: a população foi de 46 gestantes diabéticas tipo I (12\%), 45 tipo II (12\%) e 296 gestacionais (76\%). Entre as do tipo I, a cardiotocografia suspeita ou alterada correlacionou-se com Apgar de $1^{\circ}$ minuto alterado (50 e 75\%; $\left.p<0,05\right)$ e com a necessidade de UTI neonatal (50 e 75\%; $p<0,05)$. Nos casos do tipo II, a necessidade de UTI neonatal foi significativamente maior $(p<0,05)$ entre aqueles com perfil biofisico fetal alterado (67\%); a dopplervelocimetria da artéria umbilical anormal correlacionou-se com Apgar de $1^{\circ}$ minuto alterado (67\%; $\left.p<0,05\right)$. As diabéticas gestacionais com a cardiotocografia alterada apresentaram Apgar de $1^{\circ}$ minuto anormal em $36 \%$ ( $p<0,05)$, Apgar de $5^{\circ}$ minuto anormal em 18\% ( $\left.p<0,01\right)$ e óbito neonatal em $18 \%(p<0,01)$. As com indice de líquido amniótico alterado correlacionaram-se com Apgar de $5^{\circ}$ minuto anormal $(p<0,05)$ e necessidade de UTI neonatal $(p<0,05)$ e as com dopplervelocimetria da artéria umbilical alterada correlacionaram-se $(p<0,05) \mathrm{com}$ Apgar de $1^{\circ}$ e $5^{\circ}$ minuto alterado (respectivamente 25 e 8\%), necessidade de UTI neonatal (17\%) e óbito neonatal (8\%).

Conclusões: os resultados anormais nos testes de avaliação da vitalidade fetal correlacionaramse com os resultados neonatais adversos.
\end{abstract}

PALAVRAS-CHAVE: Diabetes mellitus. Cardiotocografia. Perfil biofisico fetal. Índice de líquido amniótico. Dopplervelocimetria.

Introdução

Antes do advento da insulina, as mulheres diabéticas raramente conseguiam engravidar, e quando conseguiam, eram submetidas a um risco de morte materna de cerca de $30 \%$ e de morte perinatal de $60 \%$. A partir de 1923 , com a introdução da insulina no arsenal terapêutico, a mor-

Setor de Vitalidade Fetal

Clínica Obstétrica - Hospital das Clínicas

Faculdade de Medicina da Universidade de São Paulo

Correspondência:

Roseli Mieko Yamamoto

Rua General Canavarro, 280 Bairro Campestre

09070-440 - Santo André - SP

Tel: (11) 4991-2481 / 4221-8778 FAX: (11) 4221-8752

e-mail: roselinomura@uol.com.br

Home Page: http://www.hcnet.usp.br/ob/ talidade materna se reduziu sensivelmente, a proporções inferiores a $2 \%^{1}$. Do mesmo modo a mortalidade perinatal diminuiu, principalmente devido à interação entre as equipes especializadas que atuam no acompanhamento destas pacientes.

A incidência de prematuridade é variável, podendo atingir valores de $25 \%^{2}$. Este fato se relaciona à ocorrência de descompensação do diabete durante a gravidez, desenvolvimento de polidrâmnio e de alterações na vitalidade fetal. Desta forma, a avaliação do bem-estar fetal torna-se um procedimento indispensável no acompanhamento pré-natal destas pacientes, principalmente naquelas que apresentam o diagnóstico da doença no periodo pré-gestacional.

$\mathrm{O}$ recente avanço tecnológico proporcionou 
aos obstetras a possibilidade de abordar o feto como um paciente, permitindo, com os exames disponiveis, avaliar a condição fetal, entre outros aspectos. A avaliação da vitalidade fetal constitui propedêutica fundamental ${ }^{3}$, pois, ao assegurar o bem-estar do feto, permite o prosseguimento da gestação até o termo. Assim, a utilização de propedêutica ampla pode ser necessária, na medida em que os exames mais corriqueiros se tornam insuficientes para assegurar o bemestar do feto em desenvolvimento. A avaliação da vitalidade fetal inclui vários exames como a cardiotocografia (CTG), o perfil biofísico fetal, a avaliação do volume de líquido amniótico e a dopplervelocimetria obstétrica, essenciais para a avaliação de gestações de alto risco referidas aos serviços de saúde de nível terciário ${ }^{3}$, complementados por exame ultra-sonográfico obstétrico.

O diagnóstico precoce da hipoxemia fetal propicia melhores resultados perinatais, à medida em que condutas adequadas podem ser adotadas nestas situações. O estado de descompensação diabética predispõe a maior risco de hipoxemia para o feto, sendo imprescindível o adequado controle metabólico durante a gestação para que o bem-estar fetal seja assegurado $^{4,5}$.

A Clínica Obstétrica do Hospital das Clínicas da Faculdade de Medicina da Universidade de São Paulo (HC-FMUSP), sendo um centro de referência para assistência às gestantes diabéticas, utiliza todos os métodos propedêuticos disponiveis no Setor de Vitalidade Fetal, procurando detectar precocemente o sofrimento fetal, com o objetivo primordial de reduzir a mortalidade perinatal nestas gestações ${ }^{6}$. Por outro lado, ao assegurarmos o bem-estar do concepto, fornecemos subsídios para que se permita prolongar a gestação com tranqüilidade, já que outras medidas importantes a serem adotadas demandam tempo. Indubitavelmente, o controle adequado da doença é a única alternativa para que a gestação aproxime-se do termo, quando também são maiores as chances de sucesso na realização do parto por via vaginal.

Deste modo, este estudo tem como objetivo descrever os resultados dos testes de avaliação da vitalidade fetal em gestações com diagnóstico de diabete pré-gestacional ou gestacional, analisando a relação de seus resultados com os dados neonatais.

\section{Pacientes e Métodos}

Este trabalho foi desenvolvido no Setor de
Vitalidade Fetal da Clinica Obstétrica do Hospital das Clínicas da Faculdade de Medicina da Universidade de São Paulo (HC-FMUSP). A população estudada foi selecionada retrospectivamente a partir da consulta do banco de dados do Setor. No período compreendido entre 1/11/ 91 e 31/10/96, 406 gestantes com diagnóstico de diabete foram avaliadas pelo Setor de Vitalidade Fetal. Foram excluídas as gestações gemelares, as com anomalias fetais e as pacientes cujo parto não foi realizado nesta instituição, perfazendo um total de 387 pacientes. As gestantes incluídas neste estudo foram classificadas de acordo com o tipo de diabete, perfazendo $76 \%$ (296 casos) de diabéticas gestacionais e 24\% (91 casos) de diabéticas pré-gestacionais, sendo $12 \%$ tipo I (46 casos) e $12 \%$ tipo II (45 casos).

Para a análise das freqüências dos resultados neonatais entre os grupos, foram incluídas todas as pacientes diabéticas avaliadas neste estudo, e em relação aos testes de vitalidade fetal, foram utilizados os dados referentes ao último exame da paciente antes do parto. $\mathrm{Na}$ avaliação das relações entre os exames de vitalidade fetal e os resultados neonatais, utilizamos os resultados da última avaliação realizada, no máximo, sete dias antes do parto.

O controle glicêmico foi realizado por meio da mensuração dos valores de glicemia de jejum, considerados normais os valores inferiores a $90 \mathrm{mg} / \mathrm{dL}$, e pós-prandiais de duas horas, considerados normais os valores inferiores a 120 $\mathrm{mg} / \mathrm{dL}$. As medidas adotadas para obtenção do controle glicêmico incluíram a dieta fracionada de 35 a $45 \mathrm{cal} / \mathrm{kg}$ de peso ideal por dia e a insulinoterapia, quando necessária. Iniciou-se com dose única matinal de insulina de ação lenta, que foi ajustada progressivamente no decurso da gestação, acrescentando-se doses noturnas e/ou doses pré-prandiais de insulina de ação rápida quando necessárias.

A CTG anteparto de repouso foi realizada por um período mínimo de 15 minutos, com a paciente em posição de semi-Fowler. Foram utilizados aparelhos da marca Hewlett Packard, modelos HP 8041A, com velocidade de registro gráfico de um centímetro por minuto. De acordo com o indice cardiotocométrico de Zugaib e Behle $^{7}$, o feto foi classificado como ativo (vitalidade normal), hipoativo (vitalidade suspeita) ou inativo (vitalidade alterada). Diante de um resultado suspeito ou alterado, realizou-se o teste da estimulação sônica ${ }^{7}$. O feto foi então classificado como reativo (aceleração da freqüência cardíaca fetal (FCF) de $20 \mathrm{bpm}$ por 3 minutos), hiporreativo (aceleração da FCF inferior a $20 \mathrm{bpm}$ ou a 3 minutos) ou inativo (ausência de acelera- 
ção da FCF). A resposta fetal foi classificada também como de padrão bifásico ou monofásico de acordo com a constatação ou não de acelerações transitórias após o término da resposta da FCF à estimulação sônica. De acordo com os critérios adotados neste Setor, a cardiotocografia CTG foi classificada como normal quando o feto foi ativo ou reativo, ou com padrão bifásico; suspeita quando hipoativo e hiporreativo, mantendo padrão monofásico; ou alterada quando inativo e nãoreativo.

O perfil biofísico fetal (PBF) associa o estudo das atividades biofisicas fetais, que incluem a FCF, analisada pela CTG; movimentos respiratórios fetais; movimentos corpóreos fetais e tônus fetal e a avaliação do volume de líquido amniótico. Neste estudo, utilizamos o PBF modificado (PBFm), no qual o volume de LA é analisado pela medida do índice de líquido amniótico (ILA). Os movimentos respiratórios, corpóreos e o tônus fetal foram avaliados com aparelho de ultra-sonografia da marca Toshiba SAL77B ou Diasonics SPA 1000. Durante o período máximo padronizado para observação das atividades biofísicas (30 minutos), foi caracterizada como normal a ocorrência de no mínimo 1 episódio de movimento respiratório com duração mínima de 30 segundos. Os movimentos corpóreos fetais foram classificados como normais quando o feto apresentou no mínimo 1 movimento amplo ou 3 movimentos lentos durante o período de observação, e o tônus como normal na presença de movimentos corpóreos fetais ou de acordo com os movimentos de abertura e fechamento das mãos. O ILA foi calculado de acordo com a metodologia proposta por Phelan et al., ${ }^{8}$, e o oligoidrâmnio foi caracterizado quando ILA $\leq 5,0 \mathrm{~cm}$, sendo este limite utilizado na caracterização do volume de LA anormal dentro do PBFm. O volume de LA foi ainda caracterizado como: diminuído (ILA entre 5,1 e 8,0 cm), normal (ILA entre 8,1 e $18,0 \mathrm{~cm}$ ), aumentado (ILA entre 18,1 e 25,0 $\mathrm{cm}$ ) ou polidrâmnio (ILA superior a $25,0 \mathrm{~cm})^{9}$.

Cada parâmetro do PBFm recebeu o escore 2 quando normal e o escore 0 quando anormal, sendo classificado de acordo com a sua somatória em: normal (8 ou 10), suspeito (6) ou alterado $(4,2$ ou 0$)$.

A dopplervelocimetria foi realizada por via transabdominal, utilizado-se dos seguintes equipamentos de ultra-sonografia: ATL Ultramark 9 com Doppler colorido e Diasonics SPA1000 com Doppler pulsátil. Os exames foram realizados com a paciente em posição semi-sentada. Os sonogramas dos vasos analisados foram obtidos com as imagens congeladas durante a inatividade fetal e em períodos de apnéia. Foram utiliza- dos filtros de baixa freqüência (50 hertz) e analisados sonogramas com ondas uniformes.

Para a obtenção do sonograma das artérias umbilicais, a insonação foi feita em alça livre de cordão. Quando evidenciadas velocidades diastólicas reduzidas, procurou-se analisar o cordão na porção próxima à inserção placentária. Foi calculada a relação sístole/diástole (A/ B) e os resultados foram classificados como alterados quando acima do percentil 95 da curva de normalidade adotada $^{10}$.

A avaliação dopplervelocimétrica da artéria cerebral média foi realizada em corte transverso do polo cefálico fetal, na altura dos tálamos com o transdutor deslocado obliquamente, em direção à base do crânio. Neste ponto a artéria é facilmente identificada como um ramo maior do poligono de Willis que se dirige ântero-lateralmente em direção à fissura de Sylvius. Evitou-se a compressão do abdome materno, uma vez que a compressão do polo cefálico fetal pode levar a alterações nos exames realizados nas artérias intracranianas. Para o diagnóstico de centralização da circulação fetal, os resultados foram classificados como alterados quando abaixo do percentil 5 da curva de normalidade de Arduini e Rizzo ${ }^{11}$, em idade gestacional correspondente.

Foram analisados os seguintes resultados neonatais: tipo de parto, idade gestacional no nascimento, índices de Apgar de $1^{\circ}$ e $5^{\circ}$ minuto, $\mathrm{pH}$ da artéria umbilical no nascimento, peso do recém-nascido (RN), adequação do peso do RN à idade gestacional e internação em unidade de terapia intensiva (UTI) neonatal. Os RN foram classificados como pequenos para a idade gestacional (PIG), quando com peso inferior ao percentil 10 para a idade gestacional, e como grandes para a idade gestacional (GIG), quando com peso superior ao percentil 90 , de acordo com a curva de normalidade desenvolvida pelo setor de neonatologia deste hospital ${ }^{12}$.

A idade gestacional foi calculada a partir da data da última menstruação (DUM) quando esta foi compativel com exame ultra-sonográfico realizado até a $20^{\mathrm{a}}$ semana. Quando a paciente não sabia referir a DUM ou na discordância com o exame ultra-sonográfico, a datação da gestação foi realizada com base na primeira ultra-sonografia realizada.

Foram analisadas as freqüências dos resultados dos exames e a comparação entre os grupos de estudo. Para as variáveis qualitativas utilizou-se o teste de $\chi^{2}$ com correção de Yates para continuidade, o teste de $\chi^{2}$ de MantelHaenszel e, quando pertinente, o teste exato de Fisher. Para as variáveis quantitativas, utilizouse o teste $t$ de Student. Adotou-se como nível de 
significância o valor $0,05(\alpha=5 \%)$. Com isso, níveis descritivos (p) inferiores a esse valor foram considerados significantes $(p<0,05)$.

\section{Resultados}

As características das gestantes em relação à idade, cor e paridade são mostradas na Tabela 1, na qual destacamos o predomínio da cor branca, nos três grupos analisados. A média da idade das gestantes foi significativamente diferente nos três grupos analisados. As pacientes com diabete tipo II apresentaram a maior média de idade, seguida pelo grupo com diabete gestacional, e, com menor média, o grupo com diabete tipo I. Em relação aos outros diagnósticos clínicos e/ou obstétricos associados, observamos que as sindromes hipertensivas se destacam nos três grupos, como a intercorrência mais freqüente.

\begin{tabular}{|c|c|c|c|c|c|c|}
\hline Características & $\begin{array}{l}\text { Diabete tipo I } \\
\text { (DI) } n=46\end{array}$ & $\begin{array}{l}\text { Diabete tipo II } \\
\text { (DII) } n=45\end{array}$ & $\begin{array}{l}\text { Diabete gestacional } \\
\text { (DG) } n=296\end{array}$ & DI x DII & DI x DG & DII X DG \\
\hline Idade materna em anos - média (DP) & $26,3(4,4)$ & $34,3(6,3)$ & $31,6(6,1)$ & $<0,0001$ & $<0,0001$ & $<0,01$ \\
\hline Paridade - média (DP) & $0,7(0,9)$ & $2,6(2,4)$ & $1,8(1,7)$ & $<0,0001$ & $<0,0001$ & $<0,01$ \\
\hline $\begin{array}{l}\text { Intervalo em dias entre último exame } \\
\text { e o parto - média (DP) }\end{array}$ & $1,8(2,1)$ & $2,5(3,6)$ & $3,0(5,3)$ & NS & NS & NS \\
\hline Nuliparas - $n^{0}$ de casos $(\%)$ & $23(50)$ & $8(18)$ & $58(20)$ & $<0,01$ & $<0,0001$ & NS \\
\hline Cor branca - $n^{0}$ de casos (\%) & $28(61)$ & $21(47)$ & $144(49)$ & NS & NS & NS \\
\hline $\begin{array}{l}\text { Síndrome hipertensiva associada - } \\
\mathrm{n}^{0} \text { de casos (\%) }\end{array}$ & $14(30)$ & $16(36)$ & $106(36)$ & NS & NS & NS \\
\hline
\end{tabular}

NS - não-significativo.

A distribuição dos resultados dos testes de avaliação da vitalidade fetal de acordo com o tipo de diabete está exposta na Tabela 2 . A cardiotocografia e o perfil biofísico fetal apresentaram-se normais na maioria dos casos, nos três grupos analisados. Em relação ao ILA, podemos notar que a proporção de oligoidrâmnio foi semelhante nos três grupos, e o ILA superior a $18,0 \mathrm{~cm}$ foi observado em cerca de $2,5 \%$ das pacientes com diabete tipo I e diabete gestacional. Na dopplervelocimetria da artéria umbilical, a proporção de exames alterados variou entre 10 e $17 \%$ nos grupos analisados. O estudo do fluxo da artéria cerebral média fetal foi realizado em menor número de pacientes, principalmente nos grupos de diabete tipo I e II. A proporção de resultados alterados variou de 28 a 35\%. Não foram constatadas diferenças estatisticamente significativas entre os grupos de pacientes quando analisamos a distribuição dos resultados dos testes de vitalidade fetal.

Na Tabela 3 podemos observar a distribuição dos resultados neonatais de acordo com o tipo de diabete. As pacientes com diabete tipo I apresentaram maior incidência de prematuridade, com média da idade gestacional no parto significativamente inferior à média do grupo com diabete gestacional, e proporção de partos realizados anteriormente à $37^{\text {a }}$ semana de gestação significativamente superior quando comparado aos grupos com diabete tipo II ou gestacional. O mesmo ocorreu em relação ao peso do RN, predominando, no grupo com diabete tipo I, RN de baixo peso (inferior a 2.500 gramas). A proporção de RN GIG foi significativamente maior no grupo com diabete tipo II, quando comparado às pacientes com diabete gestacional. Ao estudarmos o tipo de parto, constatamos que a freqüência de partos vaginais foi maior no grupo com diabete gestacional, sendo que a cesárea foi realizada em proporção significativamente mais alta nas pacientes com diabete tipo II quando comparadas àquelas. $\mathrm{Na}$ avaliação dos índices de Apgar, podemos observar que os RN de gestantes diabéticas tipo I e tipo II apresentaram, no primeiro minuto de vida, uma proporção significativamente maior de indice inferior a 7 quando comparados ao grupo com diabete gestacional. Do mesmo modo, a necessidade de UTI neonatal foi maior no grupo de RN de diabéticas prégestacionais. Não foram observadas diferenças significativas na ocorrência de acidose no nascimento, avaliada pelo $\mathrm{pH}$ arterial do cordão umbilical, e a detecção de mecônio no líquido amniótico também foi semelhante nos três grupos analisados. A análise da ocorrência de óbito neonatal não demonstrou diferenças significativas entre os grupos estudados. 
Tabela 2 - Distribuição dos resultados dos testes de avaliação da vitalidade fetal de acordo com o tipo de diabete.

\begin{tabular}{|c|c|c|c|c|c|c|c|}
\hline \multirow[t]{2}{*}{ Avaliação da vitalidade fetal } & \multicolumn{2}{|c|}{ Diabete tipo I (DI) } & \multicolumn{2}{|c|}{ Diabete tipo II (DII) } & \multicolumn{2}{|c|}{ Diabete gestacional (DG) } & \multirow[t]{2}{*}{$p$} \\
\hline & $\mathrm{n}$ & $(\%)$ & $\mathrm{n}$ & $(\%)$ & $\mathrm{n}$ & $(\%)$ & \\
\hline \multicolumn{8}{|l|}{ Cardiotocografia } \\
\hline Suspeita & 4 & (9) & 5 & $(12)$ & 27 & $(10)$ & NS \\
\hline Alterada & 4 & (9) & 2 & $(5)$ & 11 & (4) & \\
\hline \multicolumn{8}{|l|}{ Perfil Biofísico Fetal } \\
\hline $8-10$ & 37 & (93) & 34 & $(92)$ & 232 & $(93)$ & \\
\hline$\leq 6$ & 3 & (8) & 3 & (8) & 18 & $(7)$ & NS \\
\hline Total avaliado & 40 & & 37 & & 250 & & \\
\hline \multicolumn{8}{|l|}{ Índice de líquido amniótico } \\
\hline$>18,0 \mathrm{~cm}$ & 1 & $(2)$ & 0 & (0) & 6 & $(2)$ & \\
\hline Total avaliado & 40 & & 37 & & 253 & & \\
\hline \multicolumn{8}{|l|}{ Dopplervelocimetria } \\
\hline \multicolumn{8}{|l|}{ Artéria umbilical } \\
\hline Anormal (A/B >p95) & 4 & $(12)$ & 6 & $(17)$ & 26 & $(11)$ & NS \\
\hline Total avaliado & 34 & & 35 & & 245 & & \\
\hline \multicolumn{8}{|l|}{$\mathrm{ACM}$} \\
\hline Anormal (IP <p5) & 4 & (29) & 6 & (35) & 34 & (34) & NS \\
\hline
\end{tabular}

Tabela 3 - Resultados neonatais de acordo com o tipo de diabete.

\begin{tabular}{|c|c|c|c|c|c|c|}
\hline Resultados neonatais & $\begin{array}{l}\text { Diabete tipo I } \\
\text { (DI) } n=46\end{array}$ & $\begin{array}{l}\text { Diabete Tipo II } \\
\text { (DII) } n=45\end{array}$ & $\begin{array}{l}\text { Diabete gestacional } \\
\text { (DG) } n=296\end{array}$ & DI X DII & DI x DG & DII $x$ DG \\
\hline IG no parto - semanas, média (DP) & $36,9(2,7)$ & $37,7(2,3)$ & $38,2(1,9)$ & NS & $<0,001^{\# \#}$ & NS \\
\hline IG <37 sem no parto - $\mathrm{n}(\%)$ & $19(41)$ & $8(18)$ & $61(21)$ & $<0,05^{*}$ & $<0,01^{*}$ & NS \\
\hline Peso do RN - gramas, média (DP) & $2810,7(859,0)$ & $3107,1(783,2)$ & $3149,7(664,2)$ & NS & $<0,01^{\# \#}$ & NS \\
\hline Peso do RN <2500 g - n (\%) & $18(39)$ & $9(20)$ & $43(15)$ & $<0,05^{* *}$ & $<0,001^{*}$ & NS \\
\hline Peso do RN >4000 g - n (\%) & $3(6)$ & $5(11)$ & $20(7)$ & NS & NS & NS \\
\hline RN PIG - n (\%) & $5(11)$ & $6(13)$ & $58(20)$ & NS & NS & NS \\
\hline RN GIG - n (\%) & $6(13)$ & $11(24)$ & $31(11)$ & NS & NS & $<0,05^{*}$ \\
\hline Cesárea - n (\%) & $38(83)$ & $38(84)$ & $206(70)$ & NS & NS & $<0,05^{\star *}$ \\
\hline Apgar de $1^{\circ} \min <7-\mathrm{n}(\%)$ & $12(26)$ & $10(22)$ & $34(12)$ & NS & $<0,05^{\star}$ & $<0,05^{\star *}$ \\
\hline Apgar de $5^{\circ} \min <7-\mathrm{n}(\%)$ & $3(7)$ & $0(0)$ & $4(1)$ & NS & $<0,05^{\star *}$ & NS \\
\hline pH no nascimento <7,20 - n (\%) & $11 / 33(33)$ & $9 / 25(32)$ & $42 / 189(22)$ & NS & NS & NS \\
\hline Mecônio no LA - n (\%) & $3(7)$ & $1(2)$ & $10(3)$ & NS & NS & NS \\
\hline UTI neonatal - n (\%) & $8(17)$ & $7(16)$ & $16(5)$ & NS & $<0,01^{\#}$ & $<0,05^{\#}$ \\
\hline Óbito neonatal - n (\%) & $0(0)$ & $1(2)$ & $4(1)$ & NS & NS & NS \\
\hline
\end{tabular}

IG-idade gestacional, DP-desvio padrão, RN-recém-nascido, PIG-pequeno para idade gestacional, GIG-grande para idade gestacional, LA-líquido amniótico, UTl-unidade de terapia intensiva. * - teste do $\chi^{2},{ }^{* *}$ - teste do $\chi^{2}$ de Mantel-Haenszel, ${ }^{,}$- teste exato de Fisher, ${ }^{\#}$ - teste $t$ de Student, NS - não-significativo 
Para analisarmos as relações entre os testes de avaliação da vitalidade fetal e os resultados neonatais, estudamos apenas os casos cujo parto se deu nesta instituição até sete dias após a última avaliação fetal. Os resultados das relações com os dados neonatais, de acordo com o tipo de diabete, são mostrados nas Tabelas 4, 5 e 6 .

Tabela 4 - Resultados dos testes de vitalidade fetal de acordo com os resultados neonatais em gestantes com diabete tipo I.

\begin{tabular}{|c|c|c|c|c|c|c|c|c|c|c|c|c|}
\hline \multirow{3}{*}{$\begin{array}{l}\text { Resultados } \\
\text { neonatais }\end{array}$} & \multicolumn{3}{|c|}{ Apgar de $1^{\circ} \mathrm{min}}$. & \multicolumn{3}{|c|}{ Apgar de $5^{\circ} \mathrm{min}}$. & \multicolumn{3}{|c|}{ UTI neonatal } & \multicolumn{3}{|c|}{$\mathrm{pH}$ no nascimento } \\
\hline & $<7$ & $\geq 7$ & $p$ & $<7$ & $\geq 7$ & $p$ & $\operatorname{sim}$ & não & $p$ & $<7,20$ & $\geq 7,20$ & $p$ \\
\hline & $\mathrm{n}(\%)$ & $\mathrm{n}(\%)$ & & $\mathrm{n}(\%)$ & $n(\%)$ & & n (\%) & $\mathrm{n}(\%)$ & & $n(\%)$ & n (\%) & \\
\hline \multicolumn{13}{|l|}{ CTG } \\
\hline Normal & $7(20)$ & $28(80)$ & \multirow{3}{*}{$0,02^{*}$} & $2(6)$ & $33(94)$ & \multirow{3}{*}{ NS } & $3(9)$ & $32(91)$ & \multirow{3}{*}{$\begin{array}{l}0,002^{*} \\
0,01^{* *}\end{array}$} & $7(25)$ & $17(75)$ & \multirow{3}{*}{ NS } \\
\hline Suspeita & $2(50)$ & $2(50)$ & & $0(0)$ & $4(100)$ & & $2(50)$ & $2(50)$ & & $0(0)$ & $2(100)$ & \\
\hline Alterada & $3(75)$ & $1(25)$ & & $1(25)$ & $3(75)$ & & $3(75)$ & $1(25)$ & & $2(50)$ & $2(50)$ & \\
\hline \multicolumn{13}{|l|}{ PBF } \\
\hline $8-10$ & $11(31)$ & $25(69)$ & \multirow{2}{*}{ NS } & $3(8)$ & $33(92)$ & \multirow{2}{*}{ NS } & $6(17)$ & $30(83)$ & \multirow{2}{*}{ NS } & $8(30)$ & $19(70)$ & \multirow{2}{*}{ NS } \\
\hline$\leq 6$ & $1(33)$ & $2(67)$ & & $0(0)$ & $3(100)$ & & $2(67)$ & $1(33$ & & $0(0)$ & $2(100)$ & \\
\hline \multicolumn{13}{|l|}{ ILA } \\
\hline$\leq 5,0 \mathrm{~cm}$ & $1(20)$ & $4(80)$ & \multirow{2}{*}{ NS } & $0(0)$ & $5(100)$ & \multirow{2}{*}{ NS } & $1(20)$ & $4(80)$ & \multirow{2}{*}{ NS } & $1(25)$ & $3(75)$ & \multirow{2}{*}{ NS } \\
\hline 5,1 a $18,0 \mathrm{~cm}$ & $11(33)$ & $22(67)$ & & $3(9)$ & $30(91)$ & & $6(18)$ & $27(82)$ & & $15(46)$ & $18(54)$ & \\
\hline \multicolumn{13}{|l|}{ Doppler da AU } \\
\hline normal & $7(25)$ & $21(75)$ & \multirow{3}{*}{ NS } & $1(4)$ & $27(96)$ & \multirow{3}{*}{ NS } & $3(11)$ & $25(89)$ & \multirow{3}{*}{ NS } & $7(33)$ & $14(67)$ & \multirow{3}{*}{ NS } \\
\hline (A/B $\leq p 95)$ & & & & & & & & & & & & \\
\hline $\begin{array}{l}\text { Anormal } \\
(A / B>p 95)\end{array}$ & $1(25)$ & $3(75)$ & & $0(0)$ & $4(100)$ & & $2(50)$ & $2(50)$ & & $0(0)$ & $3(100)$ & \\
\hline
\end{tabular}

Tabela 5 - Resultados dos testes de vitalidade fetal de acordo com os resultados neonatais em gestantes com diabete tipo Il.

\begin{tabular}{|c|c|c|c|c|c|c|c|c|c|c|c|c|}
\hline \multirow{2}{*}{$\begin{array}{l}\text { Resultados } \\
\text { neonatais }\end{array}$} & \multicolumn{2}{|c|}{ Apgar de $1^{\circ} \mathrm{min}$. } & \multicolumn{4}{|c|}{ UTI neonatal } & \multicolumn{2}{|c|}{$\mathrm{pH}$ no nascimento } & \multicolumn{4}{|c|}{ Óbito neonatal } \\
\hline & $\begin{array}{c}<7 \\
\mathrm{n}(\%)\end{array}$ & $\begin{array}{c}\geq 7 \\
\mathrm{n}(\%)\end{array}$ & $p$ & $\begin{array}{c}\operatorname{sim} \\
n(\%)\end{array}$ & $\begin{array}{c}\text { não } \\
\text { n (\%) }\end{array}$ & $p$ & $\begin{array}{l}<7,20 \\
\mathrm{n}(\%)\end{array}$ & $\begin{array}{l}\geq 7,20 \\
n(\%)\end{array}$ & $p$ & $\begin{array}{c}\operatorname{sim} \\
n(\%)\end{array}$ & $\begin{array}{c}\text { não } \\
\text { n (\%) }\end{array}$ & $p$ \\
\hline \multicolumn{13}{|l|}{ CTG } \\
\hline Normal & $8(23)$ & $27(77)$ & \multirow{3}{*}{ NS } & $4(11)$ & $31(89)$ & \multirow{3}{*}{ NS } & $8(40)$ & $12(60)$ & \multirow{3}{*}{ NS } & $0(0)$ & $35(100)$ & \multirow{3}{*}{ NS } \\
\hline Suspeita & $1(25)$ & $3(75)$ & & $2(50)$ & $2(50)$ & & $1(33)$ & $2(67)$ & & $1(25)$ & $3(75)$ & \\
\hline Alterada & $0(0)$ & $2(100)$ & & $1(50)$ & $1(50)$ & & $0(0)$ & $0(0)$ & & $0(0)$ & $2(100)$ & \\
\hline \multicolumn{13}{|l|}{ PBF } \\
\hline $8-10$ & $7(22)$ & $25(78)$ & \multirow{2}{*}{ NS } & $3(9)$ & $29(91)$ & \multirow{2}{*}{$<0,05^{\#}$} & $7(37)$ & $12(63)$ & \multirow{2}{*}{ NS } & $0(0)$ & $32(100)$ & \multirow{2}{*}{ NS } \\
\hline$\leq 6$ & $1(33)$ & $2(67)$ & & $2(67)$ & $1(33)$ & & $1(33)$ & $2(67)$ & & $1(33)$ & $2(67)$ & \\
\hline \multicolumn{13}{|l|}{ ILA } \\
\hline$\leq 5,0 \mathrm{~cm}$ & $1(20)$ & $4(80)$ & \multirow{2}{*}{ NS } & $2(40)$ & $3(60)$ & \multirow{2}{*}{ NS } & $2(50)$ & $2(50)$ & \multirow{2}{*}{ NS } & $0(0)$ & $5(100)$ & \multirow{2}{*}{ NS } \\
\hline 5,1 a $18,0 \mathrm{~cm}$ & $7(23)$ & $23(77)$ & & $3(10)$ & $27(90)$ & & $6(33)$ & $12(67)$ & & $1(3)$ & $29(97)$ & \\
\hline \multicolumn{13}{|l|}{ Doppler AU } \\
\hline normal & $4(15)$ & $23(85)$ & \multirow{3}{*}{$<0,05^{\#}$} & $3(11)$ & $24(89)$ & \multirow{3}{*}{ NS } & $3(23)$ & $10(77)$ & \multirow{3}{*}{ NS } & $0(0)$ & $27(100)$ & \multirow{3}{*}{ NS } \\
\hline $\begin{array}{l}(\mathrm{A} / \mathrm{B} \leq \mathrm{p95}) \\
\text { Anormal }\end{array}$ & & & & & & & & & & & & \\
\hline $\begin{array}{l}\text { Anormal } \\
(A / B>p 95)\end{array}$ & $4(67)$ & $2(33)$ & & $3(50)$ & $3(50)$ & & $3(75)$ & $1(25)$ & & $1(20)$ & $5(80)$ & \\
\hline
\end{tabular}


Tabela 6 - Resultados dos testes de vitalidade fetal de acordo com os resultados neonatais em gestantes com diabete gestacional.

\begin{tabular}{|c|c|c|c|c|c|c|c|c|c|c|c|c|c|c|c|}
\hline \multirow{2}{*}{$\begin{array}{l}\text { Resultados } \\
\text { neonatais }\end{array}$} & \multicolumn{3}{|c|}{ Apgar de $1^{\circ} \mathrm{min}}$. & \multicolumn{3}{|c|}{ Apgar de $5^{\circ} \mathrm{min}$} & \multicolumn{3}{|c|}{ UTI neonatal } & \multicolumn{3}{|c|}{ pH no nascimento } & \multicolumn{3}{|c|}{ Óbito neonatal } \\
\hline & $\begin{array}{c}<7 \\
\mathrm{n}(\%)\end{array}$ & $\begin{array}{c}\geq 7 \\
\mathrm{n}(\%)\end{array}$ & $p$ & $\begin{array}{c}<7 \\
\mathrm{n}(\%)\end{array}$ & $\begin{array}{c}\geq 7 \\
\mathrm{n}(\%)\end{array}$ & $p$ & $\begin{array}{c}\operatorname{sim} \\
\mathrm{n}(\%)\end{array}$ & $\begin{array}{c}\text { não } \\
\text { n (\%) }\end{array}$ & $p$ & $\begin{array}{l}<7,20 \\
\mathrm{n}(\%)\end{array}$ & $\begin{array}{l}\geq 7,20 \\
\text { n (\%) }\end{array}$ & $p$ & $\begin{array}{l}\operatorname{sim} \\
\mathrm{n}(\%)\end{array}$ & $\begin{array}{c}\text { não } \\
\text { n (\%) }\end{array}$ & $p$ \\
\hline \multicolumn{16}{|l|}{ CTG } \\
\hline Normal & $24(10)$ & $204(90)$ & & $2(1)$ & $226(99)$ & & $10(4)$ & $218(96)$ & & $33(23)$ & $109(77)$ & & $2(1)$ & $226(99)$ & \\
\hline Suspeita & $3(12)$ & $21(88)$ & $<0,05^{*}$ & $0(0)$ & $24(100)$ & $<0,01^{*}$ & $1(4)$ & $23(96)$ & NS & $3(18)$ & $14(82)$ & NS & $0(0)$ & $24(100)$ & $<0,01^{*}$ \\
\hline Alterada & $4(36)$ & $7(63)$ & & $2(18)$ & $9(82)$ & & $2(18)$ & $9(82)$ & & $3(43)$ & $4(57)$ & & $2(18)$ & $9(82)$ & \\
\hline \multicolumn{16}{|l|}{ PBF } \\
\hline 8-10 & $23(10)$ & $198(90)$ & & $3(1)$ & $218(99)$ & & $10(5)$ & $211(95)$ & & $35(24)$ & $110(76)$ & & $3(1)$ & $218(99)$ & \\
\hline 6 & $1(10)$ & $9(90)$ & NS & $0(0)$ & $10(100)$ & NS & $0(0)$ & $10(100)$ & NS & $1(14)$ & $6(86)$ & NS & $0(0)$ & $10(100)$ & NS \\
\hline 4 & $2(33)$ & $4(67)$ & & $1(17)$ & $5(83)$ & & $1(17)$ & $5(83)$ & & $1(25)$ & $3(75)$ & & $1(17)$ & $5(83)$ & \\
\hline \multicolumn{16}{|l|}{ ILA } \\
\hline$\leq 5,0 \mathrm{~cm}$ & $6(15)$ & $33(85)$ & & $2(5)$ & $37(95)$ & & $4(10)$ & $35(90)$ & & $5(18)$ & $23(82)$ & & $1(3)$ & $38(97)$ & \\
\hline 5,1 a $18,0 \mathrm{~cm}$ & $18(9)$ & $176(91)$ & NS & $1(1)$ & $193(99)$ & $<0,05^{\#}$ & $6(3)$ & $188(97)$ & $<0,05^{\#}$ & $30(24)$ & $94(76)$ & NS & $2(1)$ & $192(99)$ & NS \\
\hline$>18,0$ & $2(33)$ & $4(67)$ & & $1(17)$ & $5(83)$ & & $1(17)$ & $5(83)$ & & $2(40)$ & $3(60)$ & & $1(17)$ & $5(83)$ & \\
\hline \multicolumn{16}{|l|}{ Doppler AU } \\
\hline $\begin{array}{l}\text { Normal } \\
(A / B \leq p 95)\end{array}$ & $20(10)$ & $179(90)$ & & $1(1)$ & 198 (99) & & $8(4)$ & $191(96)$ & & $27(21)$ & $99(79)$ & & $1(1)$ & $198(99)$ & \\
\hline$(A / B \leq p y s)$ & & & $<0,05^{* *}$ & & & $<0,05^{\star *}$ & & & $<0,05^{\star *}$ & & & NS & & & $<0,05^{\star *}$ \\
\hline $\begin{array}{l}\text { Anormal } \\
\text { (A/B >p95) }\end{array}$ & $6(25)$ & $18(75)$ & & $2(8)$ & $22(82)$ & & $4(17)$ & $20(83)$ & & $4(24)$ & $13(76)$ & & $2(8)$ & $22(92)$ & \\
\hline \multicolumn{16}{|l|}{ Doppler ACM } \\
\hline $\begin{array}{l}\text { Normal } \\
(\mathrm{P} \geq \mathrm{p} 5)\end{array}$ & $8(14)$ & $48(86)$ & & $1(2)$ & $55(98)$ & & $4(7)$ & $52(93)$ & & $8(19)$ & $34(81)$ & & $0(0)$ & $56(100)$ & \\
\hline $\begin{array}{l}\text { Anormal } \\
(\mathrm{IP}<\mathrm{p} 5)\end{array}$ & $7(21)$ & $26(79)$ & NS & $1(3)$ & $32(97)$ & NS & $3(9)$ & $30(91)$ & NS & $6(23)$ & $20(77)$ & NS & $2(6)$ & $31(94)$ & NS \\
\hline
\end{tabular}

CTG-cardiotocografia, PBF-perfil biofísico fetal, ILA-índice de líquido amniótico, AU-artéria umbilical, ACM-artéria cerebral média, UTI-unidade de terapia intensiva, IP-indice de pulsatilidade

${ }^{*}$ CTG (normal + suspeita) xCTG alterada - teste exato de Fisher

${ }^{* *}$ Teste exato de Fisher

\#ILA $(\leq 5,0+>18,0)$ xILA (5-18) - teste exato de Fisher

NS - não-significativo

No grupo de gestantes com diabete tipo I (Tabela 4), observamos correlação entre os resultados suspeitos ou alterados da cardiotocografia e a ocorrência de índice de Apgar de $1^{\circ}$ minuto inferior a $7 \quad(\mathrm{p}<0,05)$. A necessidade de internação em UTI neonatal também foi significativamente maior nos RN das gestantes que apresentaram um resultado suspeito ou alterado. Neste grupo não foram constatados casos que evoluíram com óbito neonatal, portanto, este parâmetro não foi analisado quanto à distribuição dos resultados dos testes de vitalidade fetal.

$\mathrm{Na}$ Tabela 5 estão os resultados neonatais de acordo com a avaliação da vitalidade fetal, nas gestantes com diabete tipo II. Observa-se associação entre o resultado do PBF inferior ou igual a 6 e a necessidade de UTI neonatal. O resultado anormal da dopplervelocimetria da artéria umbilical apresentou correlação positiva com a ocorrência de RN com índice de Apgar de $1^{\circ} \mathrm{mi}-$ nuto inferior a 7.

O estudo dos resultados neonatais de acordo com os testes de vitalidade fetal, em gestantes diabéticas gestacionais, é mostrado na Tabela 6 . O maior número de casos permitiu evidenciar com maior facilidade as relações analisadas. Podemos observar associação significativa entre a cardiotocografia alterada e a ocorrência de anormalidade nos índices de Apgar de $1^{\circ}$ e $5^{\circ}$ minuto, o mesmo ocorrendo com o óbito neonatal. $\mathrm{Na}$ análise do ILA, podemos notar que o indice diminuído ou normal (entre 5,1 e $18,0 \mathrm{~cm}$ ) apresentou menor proporção de casos com RN que apresentaram Apgar de $5^{\circ}$ minuto inferior a 7 e também menor proporção de RN internados em UTI neonatal. A proporção de óbito neonatal foi maior nos casos com ILA superior a $18,0 \mathrm{~cm}$, porém não houve diferença estatisticamente significativa, quando comparado ao grupo com ILA inferior ou igual a $18,0 \mathrm{~cm}$, ao teste exato de 
Fisher. O resultado alterado da dopplervelocimetria da artéria umbilical correlacionou-se com todos os resultados neonatais analisados na Tabela 6 , exceto a acidose no nascimento. A dopplervelocimetria da artéria cerebral média alterada não apresentou correlação com resultado neonatal adverso.

\section{Discussão}

A avaliação da vitalidade fetal tem como objetivo a detecção precoce do comprometimento da oxigenação fetal, evitando as lesões neurológicas causadas pela hipoxemia e as decorrentes da realização do parto prematuro desnecessário. O diagnóstico precoce das alterações da condição fetal reduz a ocorrência de resultados perinatais adversos, pois auxilia na ação oportuna do obstetra ao corrigir os distúrbios metabólicos que evidentemente contribuem para o aumento da mortalidade perinatal ${ }^{13}$, ou interrompendo a gestação sem os agravos da hipoxia.

Em nosso estudo, podemos observar um predominio de gestantes com diabete gestacional. As proporções de pacientes com diabete tipo I e tipo II praticamente se assemelham, incidindo em aproximadamente $12 \%$. A precocidade da doença explica o predomínio de pacientes nulíparas no grupo com diabete tipo I, acabando por influir na idade média materna, que, nestas pacientes, é inferior à observada nos outros grupos analisados. As pacientes com diabete tipo II, por sua vez, apresentam a maior média de idade, e também maior paridade, pois este tipo de diabete geralmente ocorre mais tardiamente. Salientamos a alta freqüência $(>30 \%)$ de associação com as sindromes hipertensivas, nos três grupos analisados.

Os resultados neonatais mostram que o grupo de maior gravidade, principalmente pela maior incidência de prematuridade, é o de gestantes com diabete tipo I. Estes casos geralmente apresentam maior gravidade clinica, com descontrole glicêmico mais freqüente, constituindo, ao obstetra e à equipe que acompanham estas pacientes, um desafio atingir um resultado perinatal ideal, isto é, aproximar-se ao máximo do termo. As pacientes com diabete tipo II apresentaram maior proporção de RN GIG, o que sugere ser o grupo associado ao maior descontrole glicêmico ${ }^{14}$, determinando o aumento do peso no nascimento, não-relacionado à prematuridade. Portanto, argumenta-se que estes RN se beneficiariam de um controle mais rigoroso da glicemia materna, concretizado com o uso de maior nú- mero de parâmetros na vigilância do controle metabólico na gestação. Apesar de não ser demonstrada diferença significativa, a incidência de macrossomia fetal, definida pelo peso do RN superior a $4000 \mathrm{~g}$, foi superior neste grupo de gestantes, ocorrendo em $24,4 \%$ delas. É oportuno lembrar que além dos cuidados neonatais diferenciados, decorrente da hiperinsulinemia do $\mathrm{RN}^{15}$, a assistência ao parto também merece atenção especialmente durante o período expulsivo.

$\mathrm{Na}$ análise da distribuição dos resultados dos testes de vitalidade fetal observamos, nos três grupos, a incidência de pelo menos $80 \%$ de exames cardiotocográficos normais, o que coincide com o observado em estudos já publicados anteriormente na literatura ${ }^{13,16}$. As alterações na cardiotocografia associaram-se aos parâmetros neonatais anormais no presente estudo, principalmente ao óbito neonatal no grupo com diabete gestacional, e ao índice de Apgar de $1^{\circ}$ minuto inferior a 7 nos grupos com diabete tipo I e diabete gestacional. Kjos et al. ${ }^{16}$, analisando 1.501 gestantes diabéticas, também relacionam a cardiotocografia alterada (não-reativa) com desacelerações à indicação de cesárea por sofrimento fetal.

Em relação ao índice de líquido amniótico, constatamos incidência de 12 a $15 \%$ de oligoidrâmnio (ILA $\leq 5,0 \mathrm{~cm}$ ) na população estudada, substancialmente diferente em relação ao estudo de Kjos et al. ${ }^{16}$, que relatam 3,3\% de oligoidrâmnio, definido pelo mesmo critério utilizado no presente trabalho. Estes autores não relatam a proporção de pacientes com hipertensão arterial associada, o que pode explicar as diferenças observadas nestes resultados. O estudo de Kjos et al. ${ }^{16}$ também não associa o valor do ILA com os resultados neonatais, o que no nosso trabalho foi possivel principalmente no grupo com diabete gestacional, no qual temos o maior número de pacientes. A incidência de casos com polidrâmnio é semelhante, mesmo considerando-se a diferença no critério utilizado, o que pode refletir, nos dois estudos, um predomínio de gestantes diabéticas controladas. Hipoteticamente, a análise transversal dos resultados, utilizando o ILA como variável classificatória, restringe a utilização desta metodologia na análise do volume de líquido amniótico como parâmetro do controle metabólico na gestante diabética. Existem evidências de que estudos longitudinais prospectivos bem controlados são necessários para se testar a magnitude da associação entre as variações no ILA e a adequação do controle glicêmico na gestante diabética ${ }^{5}$, principalmente nas usuárias de insulinoterapia.

As alterações na dopplervelocimetria da 
artéria umbilical refletem aumento de resistência ao fluxo sangüíneo, representando uma anormalidade na circulação feto-placentária ${ }^{17}$. Nesta situação de falência placentária há prejuízo nas trocas materno-fetais, culminando com a ocorrência de resultados neonatais adversos. No presente estudo, em que pese a pequena freqüência de resultados anormais, podemos notar maior incidência de RN com índices de Apgar de $1^{\circ}$ e $5^{\circ}$ minuto inferior a 7 , maior necessidade de UTI neonatal e maior proporção de óbito neonatal no grupo com Doppler de artéria umbilical anormal, principalmente nas gestantes com diabete gestacional, cujo número total de pacientes é superior ao dos outros dois grupos analisados.

A dopplervelocimetria da artéria cerebral média foi realizada em maior número de pacientes no grupo de diabete gestacional, e nestas não observamos correlação entre a sua anormalidade e os resultados perinatais adversos, tal como tem sido descrito em poucos trabalhos da literatura $^{18}$. Temos estudado principalmente o índice de pulsatilidade e relacionado somente com o resultado neonatal, porém novas perspectivas despontam no estudo da velocidade sistólica neste vaso, principalmente no que diz respeito à correlação com o controle metabólico da gestante diabética. Novos protocolos em andamento em nosso Setor objetivam estabelecer as relações entre estes novos parâmetros.

Nas últimas duas décadas, diversos protocolos têm sido propostos para avaliação antenatal do bem-estar fetal em gestantes com diabete melito gestacional ou pré-gestacional ${ }^{19,20}$. Na Clínica Obstétrica da FMUSP, o Setor de Endocrinopatias existente há mais de 20 anos tem acumulado ampla experiência no controle da gestante diabética, conseguindo inegável sucesso neste compromisso. Ao longo deste periodo, várias modificações foram introduzidas no protocolo assistencial, notadamente na manipulação da terapêutica insulínica, destacando-se a habilidade e o refinamento no ajuste de suas doses de acordo com a monitorização rigorosa do controle glicêmico.

O trabalho conjunto com o Setor de Vitalidade Fetal permite ao pré-natalista especializado no controle de gestantes diabéticas aguardar com segurança que o feto atinja a maturidade, antes de se optar pela resolução prematura da gestação. O nosso Setor tem obtido sucesso no acompanhamento das gestantes diabéticas, utilizando os exames analisados no presente trabalho. As alterações observadas neles (cardiotocografia, o perfil biofísico fetal, o indice de liquido amniótico e a dopplervelocimetria da artéria umbilical) se correlacionaram com resultados neonatais adversos, comprovando a necessidade da monitorização do bem-estar fetal nas gestantes diabéticas. Assim são identificados os casos de maior risco de um resultado desfavorável, reconhecendo aqueles que realmente necessitam da resolução imediata da gestação. O presente estudo demonstra a importância da aplicação de uma propedêutica abrangente do bemestar fetal, pois diferentes comportamentos foram observados nos três grupos.

\section{SUMMARY}

Purpose: to study the fetal well-being assessment in pregnancies complicated by diabetes, and to analyze the neonatal results.

Methods: we studied 387 pregnant women with diabetes at the Fetal Surveillance Unit. The last examination (cardiotocography, fetal biophysical profile, amniotic fluid index and dopplervelocimetry) was correlated with the neonatal outcome.

Results: the studied population included 46 (12\%) type I diabetes, 45 (12\%) type II and 296 (76\%) gestational diabetes. Type I diabetes with abnormal or suspected cardiotocography was related to abnormal 1st minute Apgar (50 and 75\%, $p<0.05)$ and to the need for neonatal intensive care unit (50 and $75 \%, p<0.05)$. The abnormal biophysical profile in type II diabetic pregnancy was related to the need for neonatal intensive care $167 \%$, $p<0.05)$, and abnormal umbilical artery Doppler study was related to abnormal 1st minute Apgar (67\%, $p<0.05)$. Gestational diabetes with abnormal cardiotocography presented 36\% abnormal 1st minute Apgar $(p<0.05)$, 18\% abnormal 5th minute Apgar $(p<0.01)$ and $18 \%$ neonatal death $(p<0.01)$. Abnormal amniotic fluid index was related to abnormal 5th minute Apgar $(p<0.05)$ and need for neonatal intensive care unit $(p<0.05)$. Gestational diabetes with abnormal umbilical artery Doppler was related $(p<0.05)$ to: abnormal 1 st and $5^{\text {th }}$ minute Apgar, respectively, 25 and $8 \%$, Need for neonatal intensive care in $17 \%$ and neonatal death in $8 \%$.

Conclusions: the fetal well-being examinations correlated with adverse perinatal outcome, showing the need for fetal surveillance in diabetic pregnant women.

KEY WORDS: Diabetes mellitus. Cardiotocography. Fetal biophysical profile. Amniotic fluid index. Dopplervelocimetry. 


\section{Referências:}

1. Carrington ER. Diabetes in pregnancy. Clin Obstet Gynecol 1973; 16:28-46.

2. Gabbe SG. Management of diabetes mellitus in pregnancy. Am J Obstet Gynecol 1985; 153:8248.

3. Black RS, Campbell S. Cardiotocography versus Doppler. Ultrasound Obstet Gynecol 1997; 9:14851.

4. Landon MB, Gabbe SG. Fetal surveillance in the pregnancy complicated by diabetes mellitus. Clin Obstet Gynecol 1991; 34:535-43.

5. Dashe JS, Nathan L, McIntire DD, Leveno KJ. Correlation between amniotic fluid glucose concentration and amniotic fluid volume in pregnancy complicated by diabetes. Am J Obstet Gynecol 2000; 182:901-4.

6. Miyadahira S. Vitalidade fetal. In: Zugaib M, Bittar RE, editores. Protocolos Assistenciais da Clínica Obstétrica da Faculdade de Medicina da USP. $1^{\mathrm{a}}$ ed. São Paulo: Atheneu; 1997. p.36-48.

7. Zugaib M, Behle I. Monitoração fetal eletrônica. $1^{\text {a }}$ ed. São Paulo: Roca; 1981. p.55-78.

8. Phelan JP, Smith CV, Broussard P, Small M. Amniotic fluid volume assessment with the fourquadrant technique at 36-42 weeks' gestation. J Reprod Med 1987; 32:540-2.

9. Yamamoto RM, Miyadahira S, Zugaib M. Avaliação do volume do líquido amniótico: diagnóstico ultrasonográfico e conduta no oligoidrâmnio e poliidrâmnio. Rev Ginecol Obstet 1996; 7:25-32.

10.Amim Júnior J, Lima MLA, Fonseca ALA, Chaves Netto $\mathrm{H}$, Montenegro CAB. Dopplerfluxometria da artéria umbilical: valores normais para a relação $A / B$, indice de resistência e índice pulsátil. J Bras Ginecol 1990; 100:337-49.

11.Arduini D, Rizzo G. Normal values of Pulsatility Index from fetal vessels: a cross-sectional study on 1556 healthy fetuses. J Perinat Med 1990; 18:165-72.
12. Ramos JLA. Avaliação do crescimento intra-uterino por medidas antropométricas do recém-nascido [tese]. São Paulo: Universidade de São Paulo; 1983.

13.Golde SH, Montoro M, Good-Anderson B, et al. The role of nonstress tests, fetal biophysical profile, and contraction stress tests in the outpatient management of insulin-requiring diabetic pregnancies. Am J Obstet Gynecol 1984; 148:269-73.

14.Jovanovic L, Peterson CM, Saxena BB, Dawood MY, Saudek CD. Feasibility of maintaining normal glucose profiles in insulin-dependent pregnant diabetic women. Am J Med 1980; 68:105-12.

15.Harman CR, Menticoglou SM. Fetal surveillance in diabetic pregnancy. Curr Opin Obstet Gynecol 1997; 9:83-90.

16.Kjos SL, Leung A, Henry OA, Victor MR, Paul RH, Medearis AL. Antepartum surveillance in diabetic pregnancies: predictors of fetal distress in labor. Am J Obstet Gynecol 1995; 173:15329.

17.Giles WB, Trudinger BJ, Baird PJ. Fetal umbilical artery flow velocity waveforms and placental resistance: pathological correlation. $\mathrm{Br} \mathrm{J}$ Obstet Gynaecol 1985; 92:31-8.

18.Poniedzialek-Czajkowska E, Leszczynska-Gorzelak B, Sawatynski A, Oleszczuk J. The value of Doppler velocimetry of umbilical and middle cerebral arteries in pregnancy complicated by diabetes mellitus. Ginekol Pol 1999; 70:672-8.

19.Lagrew DC, Pircon RA, Towers CV, Dorchester W, Freeman RK. Antepartum fetal surveillance in patients with diabetes: when to start? Am J Obstet Gynecol 1993; 168:1820-6.

20.Banduki Neto JD. Diabete melito. In: Zugaib M, Bittar RE, editores. Protocolos Assistenciais da Clínica Obstétrica da Faculdade de Medicina da USP. $1^{a}$ ed. São Paulo: Atheneu; 1997. p.112-7. 